\title{
Reduction of Vibration of Fluid Loaded Plate with Piezoelectric Elements - a Comparison of Analytical, FEM Harmonic Analysis, and Real Results
}

\author{
D. IWAŃSKI* AND J. WICIAK \\ AGH - University of Science and Technology, Faculty of Mechanical Engineering and Robotics \\ Department of Mechanics and Vibroacoustics, al. A. Mickiewicza 30, 30-059 Krakow, Poland
}

\begin{abstract}
In the present paper, a comparison of analytical calculations, FEM harmonic analysis, and real data from laboratory stand of fluid-loaded structure were taken under consideration. Resonant frequencies of the investigated structure were measured using swept sine technique on laboratory stand. Analytical calculations of the first twelve modes were made and the numerical model was build using the finite element method. Results from real data, analytical calculations, and numerical calculations are comparable.
\end{abstract}

DOI: 10.12693 /APhysPolA.123.1040

PACS: 43.28.Js, 43.30.-k, 43.30.+m

\section{Introduction}

Current technology provides better access to smart materials which can help to analyze and reduce vibration and noise in the surrounding environment $[1,2]$. There were several attempts to give theoretical description of vibration of a structure composed of a beam and a piezoelectric transducer $[3,4]$ or a plate and a piezoelement of arbitrary shape $[5,6]$. Analytical solutions of appropriate problems are usually obtained using the Fourier method and a distribution of piezoelectric transducers is possible to be found analytically [7]. Despite many applications of smart materials in various fields of technology [8], there are just few among them regarding the use in the aquatic environment $[9,10]$.

\section{Methodology of research}

\subsection{Description of the system}

The subject of analysis is the electrolytically coated non-alloy steel circular plate (PN-EN 10335) with diameter of $150 \mathrm{~mm}$ and thickness of $0.4 \mathrm{~mm}$ bounded around its circumference. One of the sides of the circular plate contains eight $20 \mathrm{~mm} 20 \mathrm{~mm} \times 1 \mathrm{~mm}$ Sumida PZT 4D piezoelements arranged in two concentric circles (see Fig. 1a). The opposite side of the plate is loaded with fluid from the water tank attached to the plate (see Fig. 1b). Two cases of load on the plate were taken under consideration: the plate loaded with fluid and the plate loaded with air. One of the piezoelements attached to the plate was used as a source of sound and the other as sensors. For such prepared design the following analyses were made: harmonic response of real structure using

*corresponding author; e-mail: iwanski@agh.edu.pl swept sine technique, modal and harmonic analyses using finite element method (FEM) and analytical calculations of natural frequencies of a clamped circular plate. Due to axisymmetric structure resulting in similar response between inner and outer piezoelements, one of the piezoelement was chosen as an actuator (see Fig. 1a).
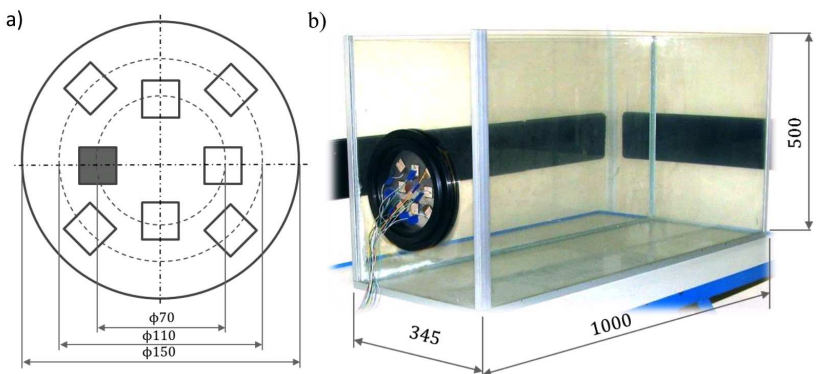

Fig. 1. (a) Piezoelements arrangement (on inner and outer circle) of the analyzed structure with dark one marked as an actuator; (b) laboratory stand (water tank). Dimensions in millimeters.

\subsection{Analytical solution}

The plate was clamped around its circumference hence simple analytical calculations of natural frequencies were made for unloaded plate [11]

$$
\omega_{m n}=\left(\frac{\lambda_{m n}^{2}}{r_{0}^{2}}\right) \sqrt{\frac{\rho}{D h}}
$$

and for fluid loaded plate [12]

$$
\omega_{F m n}=\frac{\omega_{m n}}{\sqrt{1+\Gamma_{m n}\left(\frac{\rho_{F}}{\rho_{P}}\right)\left(\frac{r}{h}\right)}},
$$




\begin{tabular}{l|l}
\hline \multicolumn{1}{c|}{ Parameter } & $\begin{array}{l}\text { Value used in } \\
\text { calculations }\end{array}$ \\
\hline $\mathrm{m}, \mathrm{n}-$ number of modes & $0,1,2,3, \ldots$ \\
\hline$r_{0}-$ radius of the plate boundary $[\mathrm{m}]$ & 0.075 \\
\hline$D-$ rigidity of the plate $[\mathrm{N}]$ & 1.86 \\
\hline$E-$ Young's modulus $[\mathrm{Pa}]$ & $2.1 \times 10^{11}$ \\
\hline$h-$ thickness of the plate $[\mathrm{m}]$ & 0.00046 \\
\hline$\nu-$ Poisson's ratio $\left[\mathrm{kg} / \mathrm{m}^{3}\right]$ & 0.29 \\
\hline$\rho-$ density of the material of the & 7748 \\
plate $\left[\mathrm{kg} / \mathrm{m}^{3}\right]$ & \\
\hline$\rho_{F}-$ density of the water $\left[\mathrm{kg} / \mathrm{m}^{3}\right]$ & 1000 \\
\hline$\rho_{P}-$ density of the air $\left[\mathrm{kg} / \mathrm{m}^{3}\right]$ & 1.225 \\
\hline$\lambda-$ ratio & $10.2158,21.3$, \\
& $34.9, \ldots$ \\
\hline$\Gamma_{m n}-\quad$ NAVMI (non-dimensional & $208.8,434.5$, \\
added virtual mass incremental) & $712.9, \ldots$ \\
& 2334.4
\end{tabular}

\subsection{FEM model}

Both the plate and the piezoelements were modeled with respect to proper boundary conditions (see Fig. 2). The modal and the harmonic analysis using FEM were performed in the Ansys software environment. Numerical model was build using following types of element: for the plate - Solsh190 (Solid-shell), for piezoelements Solid226, and for fluid - Fluid30 and Fluid130. Piezoelectric layers were modeled by two layers of finite elements. Structural parts were divided into $0.004 \mathrm{~mm}$ length elements and fluid part into $0.02 \mathrm{~mm}$ length elements. All elements were connected by nodes. This size was taken considering discrepancy of the results (see Fig. 3) and calculations time.
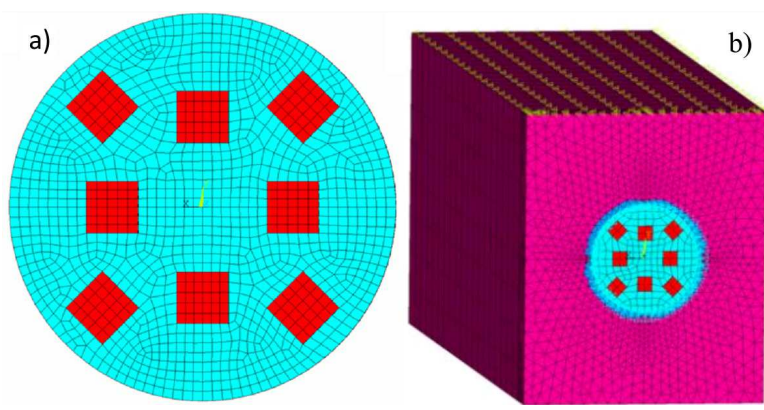

Fig. 2. Mesh and boundary conditions of analyzed model: (a) circular plate with piezoelements; (b) plate loaded with water.

\subsection{Measurement stand}

In order to get harmonic response of the investigated structure, appropriate measurement chain was set up (see Fig. 4). Resonant frequencies were found by swept sine analysis using National Instruments PCI 6230 card and

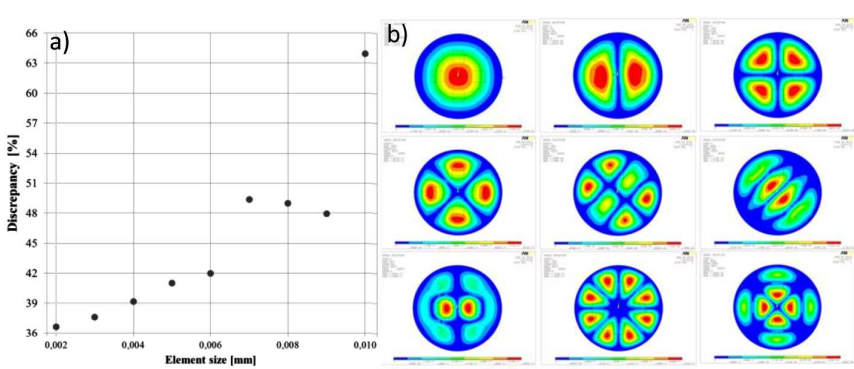

Fig. 3. (a) Discrepancy of the numerical results for different element size in modal analysis; (b) numerical results from modal analysis of first 9 modes.

PC with LabView software. This signal was amplified to about $50 \mathrm{~V}$ using Apart MB150 amplifier. Remaining piezoelements attached to the plate acted as sensors giving more precise but comparable results.

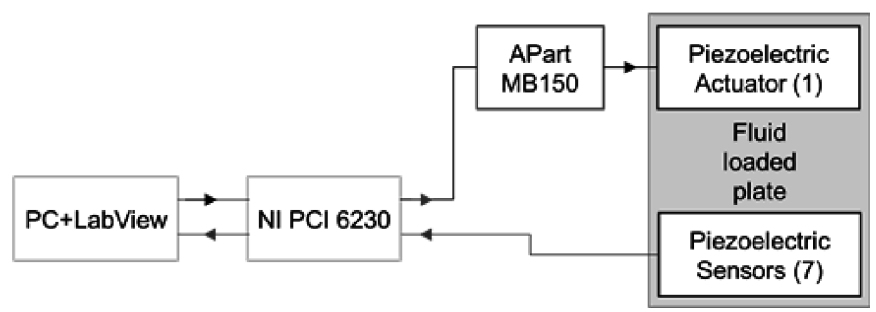

Fig. 4. Schematic diagram of the measurement chain.

The measurement on the laboratory stand was performed to get the frequency response of the system (see Fig. 5). All seven channels (one used as actuator) produced comparable results in an amplitude and frequency domain. This confirms that the measurement has been performed correctly.

There are significant differences between the results of the real system response for air-loaded and fluid-loaded plate. As expected (Eq. (2)), single-sided fluid loading shifts the resonant frequency of the plate. For the first six modes, the shift is almost doubled.

Next, the FEM analyses using Ansys software were performed. The first was the modal analysis of the fluidloaded clamped plate with piezoelements (Fig 4a,b).

One may notice that the element size does influence significantly the results, i.e. the frequency modes. However, decreasing element size generates exponential growth of the calculation time.

The second one was the harmonic analysis for frequency range from 1 to $1100 \mathrm{~Hz}$. Results compared with real data are shown in Fig. 6 and in Table I. For airloaded plate, the numerical model and analytical calculations are almost equal with discrepancy less than $0.08 \%$. When comparing real data with numerical results one can observe still very good results with discrepancy less than $6 \%$. As for the fluid loaded plate the numerical model gives less accurate results. When comparing both real and numerical model, the discrepancy is within $37 \%$ 
TABLE I

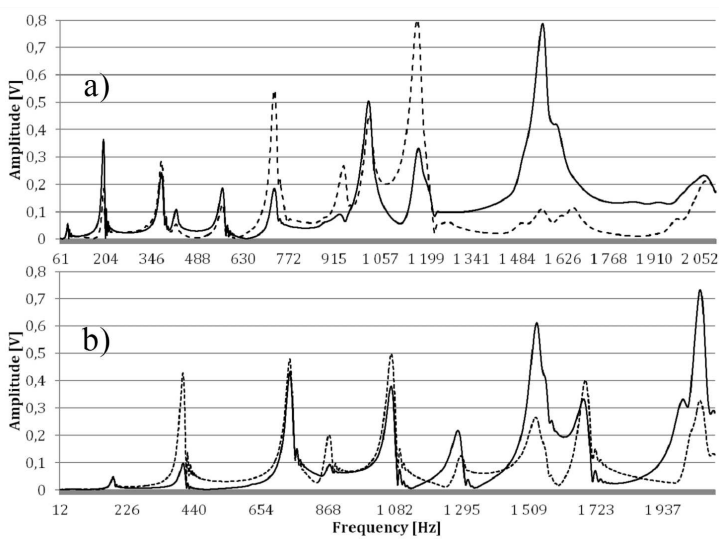

Fig. 5. Frequency response of the structure for plate loaded with fluid: (a) air; (b) water; using the two most representative channels from all having been measured.

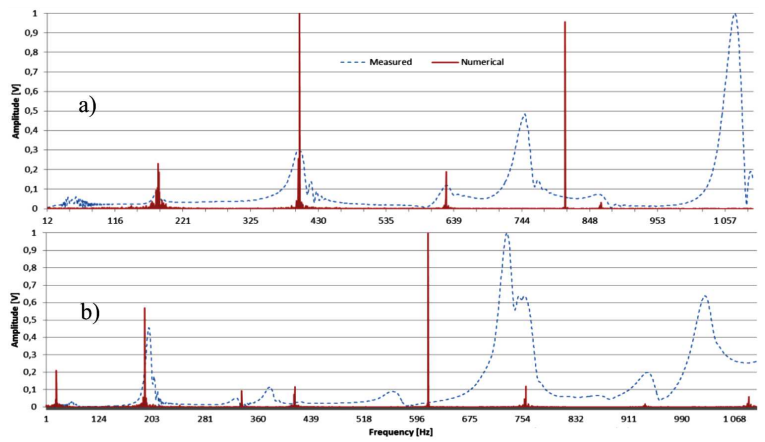

Fig. 6. Frequency response of the structure (dotted line) and numerical results (solid line) for plate loaded with fluid: (a) air; (b) water.

and for numerical and analytical — within 18\%. These differences arise from the adopted and simplified numerical model which does not reflect all complex elements of the real structure. However when compared to other work [4] these results have less errors, mostly due to element size, connection (by nodes instead of equations) and the plate element type. What is more, more modes have been fitted to real results.

Due to the high value of the discrepancy additional test was performed - the correlation analysis of amplitudes and phases of the piezoelements voltage. Both real and modal analysis results were properly scaled to unity and then the correlation analysis was performed using Statistica software. The goal function was to find statistically significant correlation $r$ so that $|r|>0.8$ (see Table II). The results in the table, in the most cases meet the required condition so for the authors the numerical model is accurate.

\section{Conclusions}

- Numerical calculations are always different from the real results. Increasing element size produces less errors but needs more calculation time.
The first twelve natural frequencies of the investigated structure compared with analytical and numerical results.

\begin{tabular}{c|c|c|c|c|c|c}
\hline \hline$\omega_{m n}[\mathrm{~Hz}]$ & \multicolumn{3}{|c|}{ Results for air } & \multicolumn{3}{c}{ Results for water } \\
\hline mode & real & analyt. & ansys & real & analyt. & ansys \\
\hline 0.0 & 182.0 & 181.6 & 181.6 & 84.2 & 53.3 & 59.0 \\
\hline 1.0 & 401.0 & 377.9 & 377.9 & 197.9 & 158 & 192.2 \\
\hline 2.0 & 627.6 & 620.1 & 620.0 & 327.4 & 268.2 & - \\
\hline 0.1 & 748.0 & 707.1 & 706.9 & 389.6 & 344.1 & 336.3 \\
\hline 3.0 & 861.8 & 907.5 & 907.2 & 422.3 & 479.9 & 414.2 \\
\hline 1.1 & 1071.9 & 1081.9 & 1081.4 & 559.5 & 577.3 & 612.4 \\
\hline 4.0 & 1287.4 & 1239.3 & 1238.7 & 729.8 & 664.2 & 758.3 \\
\hline 2.1 & 1550.5 & 1504.9 & 1504.0 & 938.5 & 865.9 & 935.8 \\
\hline 0.2 & 1581.5 & 1585.3 & 1584.3 & 944.0 & 922.7 & 964.6 \\
\hline 5.0 & 1692.7 & 1614.6 & 1613.5 & 1024.2 & 982.8 & 1008.3 \\
\hline 3.1 & 2014.1 & 1975.9 & 1974.5 & 1171.4 & 1204.2 & 1089.2 \\
\hline 6.0 & 2042.2 & 2032.8 & 2031.2 & 1223.7 & 1261.5 & 1192.9 \\
\hline \multirow{2}{*}{ max. discrepancy } & re.-ana. & re.-ans. & & re.-ana. & re.-ans. \\
& & $<6 \%$ & $<6 \%$ & & $<37 \%$ & $<30 \%$ \\
\cline { 2 - 7 } & \multicolumn{2}{|c|}{ ans.-ana. } & & ans.-ana. \\
\cline { 2 - 7 } & \multicolumn{2}{|c|}{$\ll 1 \%$} & & \multicolumn{2}{c}{$<18 \%$}
\end{tabular}

TABLE II

Results from correlation analysis of real and numerical calculations of the piezoelements voltage.

\begin{tabular}{c|c|c}
\hline \hline Numerical result [Hz] & Real data [Hz] & Correlation $r$ \\
\hline 59 & 88 & 0.86 \\
194 & 202 & 0.94 \\
333 & 310 & 0.81 \\
336 & 328 & -0.79 \\
402 & 375 & 0.96 \\
413 & 419 & 0.88 \\
616 & 553 & 0.85 \\
762 & 718 & -0.96 \\
788 & 741 & 0.98 \\
933 & 953 & -0.83 \\
941 & 954 & -0.95 \\
1065 & 1021 & 0.83 \\
1088 & 1052 & -0.81
\end{tabular}

- There are significant differences between resonant frequencies in air loaded and water loaded plate.

- Discrepancy between results from numerical model and air loaded plate reveals very good conformity and the goal function is achieved.

- Numerical model for fluid-loaded plate reveals good agreement with measurement results but needs further investigation.

- In modal analysis when using piezoelements a good way of confirmation of the results, i.e. frequency modes with real data, is the correlation analysis of the amplitudes and phases of the piezoelement voltages. 


\section{Acknowledgments}

The work was carried out using a grant no. N N504 670640

\section{References}

[1] E.F. Crawley, J. de Luis, Am. Inst. Aeronautics Astronautics J. 25, 1273 (1987).

[2] A. Donoso, J.C. Bellido, J. Sound Vibr. 319, 50 (2009).

[3] C.R. Fuller, S.J. Elliot, P.A. Nelson, Active Vibration Control, Academic Press Limited, London 1996.

[4] A. Brański, Acta Phys. Polon. A 121, A-126 (2012).
[5] E.K. Dimitriadis, C.R. Fuller, C.A. Rogers, J. Vib. Acoust 113, 100 (1991).

[6] M. Wiciak, Acta Phys. Polon. A 121, A-142 (2012).

[7] A. Brański, G. Lipinski, Acta Phys. Polon. A 119, 936 (2011).

[8] M.S. Kozień, J. Wiciak, Archiv. Acoust. 33, 643 (2008).

[9] J. Wiciak, Archiv. Acoust. 32, suppl 265 (2007).

[10] L. Leniowska, Mechanics 27, 18 (2008).

[11] S. Kaliski, Technical Mechanics, vol. III - vibrations and waves, PWN, Warszawa 1986.

[12] C.C. Liang, Y.S. Tai, P.L. Li, J. Sound Vibr. 228, 1167 (1999). 